\title{
Cell-autonomous programming of rat adipose tissue insulin signalling proteins by maternal nutrition
}

\author{
Malgorzata S. Martin-Gronert ${ }^{1} \cdot$ Denise S. Fernandez-Twinn ${ }^{1} \cdot$ Martin Bushell $^{2}$ • \\ Kenneth Siddle $^{1}$ • Susan E. Ozanne ${ }^{1}$
}

Received: 14 October 2015 / Accepted: 3 February 2016 / Published online: 10 March 2016

(C) The Author(s) 2016. This article is published with open access at Springerlink.com

\begin{abstract}
Aims/hypothesis Individuals with a low birthweight have an increased risk of developing type 2 diabetes mellitus in adulthood. This is associated with peripheral insulin resistance. Here, we aimed to determine whether changes in insulin signalling proteins in white adipose tissue (WAT) can be detected prior to the onset of impaired glucose tolerance, determine whether these changes are cell-autonomous and identify the underlying mechanisms involved.

Methods Fourteen-month-old male rat offspring born to dams fed a standard protein $(20 \%)$ diet or a low $(8 \%)$ protein diet throughout gestation and lactation were studied. Fat distribution and adipocyte size were determined. Protein content and mRNA expression of key insulin signalling molecules were analysed in epididymal WAT and in pre-adipocytes that had undergone in vitro differentiation.

Results The offspring of low protein fed dams (LP offspring) had reduced visceral WAT mass, altered fat distribution and a higher percentage of small adipocytes in epididymal WAT. This was associated with reduced levels of IRS1, PI3K p110 $\beta$, Akt1 and PKC $\zeta$ proteins and of phospho-Akt Ser473. Corresponding mRNA transcript levels were unchanged. Similarly, in vitro differentiated
\end{abstract}

Malgorzata S. Martin-Gronert

msm32@cam.ac.uk

1 University of Cambridge Metabolic Research Laboratories and MRC Metabolic Diseases Unit, Wellcome Trust-MRC Institute of Metabolic Science, Addenbrooke's Hospital, Box 289, Cambridge CB2 OQQ, UK

2 MRC Toxicology Unit, University of Leicester, Hodgkin Building, Leicester, UK adipocytes from LP offspring showed reduced protein levels of IR $\beta$, IRS1, PI3K $\mathrm{p} 85 \alpha$ and $\mathrm{p} 110 \beta$ subunits, and Akt1. Levels of Akt Ser473 and IRS1 Tyr612 phosphorylation were reduced, while IRS1 Ser307 phosphorylation was increased.

Conclusions/interpretation Maternal protein restriction during gestation and lactation changes the distribution and morphology of WAT and reduces the levels of key insulin signalling proteins in the male offspring. This phenotype is retained in in vitro differentiated adipocytes, suggesting that programming occurs via cell-autonomous mechanism(s).

Keywords Adipose tissue - Cell-autonomous mechanisms · Developmental programming · Insulin signalling $\cdot$ Low birthweight $\cdot$ Maternal diet $\cdot$ Protein restriction - Type 2 diabetes

$\begin{array}{ll}\text { Abbreviations } & \\ \text { ERK } & \text { Extracellular signal-regulated kinase } \\ \text { GLUT4 } & \text { Glucose transporter type 4 } \\ \text { IR } \beta & \text { Insulin receptor beta } \\ \text { JNK } & \text { c-Jun N-terminal kinase } \\ \text { LBW } & \text { Low birthweight } \\ \text { LP offspring } & \text { Offspring of low protein fed dams } \\ \text { MAPK } & \text { Mitogen-activated protein kinase } \\ \text { MiRNA } & \text { microRNA } \\ \text { PI3K } & \text { Phosphatidylinositol 3-kinase } \\ \text { PKC } ~ & \text { Protein kinase C } ~ \\ \text { qRT-PCR } & \text { Quantitative RT-PCR } \\ \text { SOCS1 } & \text { Suppressor of cytokine signalling 1 } \\ \text { UTR } & \text { Untranslated region } \\ \text { WAT } & \text { White adipose tissue }\end{array}$




\section{Introduction}

Suboptimal nutrition during fetal and early postnatal life can have profound and lifelong detrimental effects on the health of an individual. Undernutrition in fetal life, which leads to intrauterine growth restriction (IUGR) and a low birthweight (LBW), is associated with an increased risk of developing multiorgan metabolic pathologies such as type 2 diabetes mellitus and the metabolic syndrome. The molecular mechanisms through which early nutrition permanently alters the phenotype of an organism and programmes its long-term metabolic health are slowly becoming defined.

Animal models have proven invaluable for studying the mechanisms linking early nutrition and type 2 diabetes risk [1]. In this context, we and others have used a rat model of maternal protein restriction. The offspring of low protein fed dams (LP offspring) share phenotypic similarities with LBW humans, including the development of insulin resistance in both skeletal muscle and white adipose tissue (WAT) and age-dependent loss of glucose tolerance that lead to the development of type 2 diabetes [2,3]. Prior to the development of whole body insulin resistance, downregulation of key insulin signalling proteins such as insulin receptor substrate-1 (IRS1), glucose transporter type 4 (GLUT4), and the p110 $\beta$ catalytic subunit and $\mathrm{p} 85 \alpha$ regulatory subunit of phosphatidylinositol 3-kinase (PI3K) have been observed in skeletal muscle and WAT of both LBW men and LP offspring rats [2, 4-6]. These changes were detected in 15-month-old LP offspring rats, which had already developed impaired glucose tolerance.

Changes in the levels of key insulin signalling proteins were not accompanied by alterations in the levels of corresponding mRNAs, indicating that post-transcriptional regulatory mechanism(s) are involved. Recently, microRNAs (miRNAs) have emerged as important programming factors that can regulate gene expression by modulating mRNA translation without altering mRNA levels [7, 8]. Other post-transcriptional regulatory mechanisms such as protein degradation have also been implicated in pathogenesis of type 2 diabetes [9].

In recent years, our understanding of the importance of WAT in regulating whole body metabolic homeostasis has increased. WAT is the predominant type of fat in adult humans and rodents. It not only acts as the principal storage site for lipids but also secretes adipose-derived factors (i.e. adipokines) that can affect the metabolism of other organs and tissues [10, 11]. WAT appears particularly vulnerable to suboptimal early nutrition: some of the earliest and most substantial effects of early nutritional manipulation have been observed in this tissue $[12,13]$. The aims of the current study were to: (1) investigate whether changes in insulin signalling molecules in the WAT of rats exposed to a low protein diet in utero and during the suckling period are present before the development of impaired glucose tolerance; (2) determine whether these changes are cell-autonomous; and (3) further investigate the potential mechanisms involved, focusing on post-transcriptional gene regulatory mechanisms.

\section{Methods}

Animals All studies were approved by the University of Cambridge Animal Welfare and Ethical Review Board, and conducted according to the Home Office Animals (Scientific Procedures, UK) Act 1986. Stock Wistar Han rats (Rattus norvegicus; Crl:WI[Han] strain) were purchased from Charles River (Tranent, UK) and dams were produced by inhouse breeding of stock animals. Virgin female rats weighing 240-260 g were individually housed in a specific pathogen free (SPF) environment in individually ventilated cages with environmental enrichment and maintained at $22^{\circ} \mathrm{C}$ on a $12 \mathrm{~h}$ light:dark cycle. After mating, the day on which virginal plugs were expelled was taken as day 1 of gestation. Pregnant dams were given ad libitum access to control (20\% protein, wt/vol.) or an isoenergetic low protein ( $8 \%$ protein, wt/vol.) diet [14]. At $48 \mathrm{~h}$ after delivery, litters were reduced at random to eight pups: four males and four females. Lactating dams were maintained on experimental diets. Twenty-one-day-old pups were weaned onto standard chow (LAD1 diet, Special Diets Services, Witham, UK), fed ad libitum and maintained in pairs in SPF cages with environmental enrichment. Two 14-monthold males from each litter were fasted overnight. The next day, their body weights were recorded, blood was taken for glucose measurement and plasma was obtained for biochemical analysis. The rats were then killed by $\mathrm{CO}_{2}$ asphyxiation, and individual fat depots were collected and weighed. The intraabdominal (mesenteric) fat depot was collected from around the intestines, retroperitoneal (perirenal) fat was collected from around the kidneys and epididymal (gonadal) fat was collected from near the testes. WAT samples were snapfrozen and stored at $-80^{\circ} \mathrm{C}$.

Biochemical analysis Fasting glucose concentrations were measured in tail blood using a blood glucose analyser (AlphaTRAK, Abbott, Maidenhead, UK)). Fasting plasma insulin and leptin concentrations were determined by ELISA (ultrasensitive rat insulin EIA, Mercodia, Uppsala, Sweden; rat leptin ELISA, Crystal Chem, Downers Grove, IL, USA) and lipid content was measured using a Folch assay [15]. Lipid analysis was performed by the Core Biochemical Assay Laboratory (Cambridge, UK).

Analysis of adipocyte cell size and number Epididymal WAT was fixed in paraformaldehyde, processed on a 14-h cycle (VIP6, Sakula, Torrance, CA, USA), embedded in paraffin, cut into $5 \mu \mathrm{m}$ sections, placed onto Superfrost microscope slides (VWR, Lutterworth, UK) using synthetic mountant (Thermo Scientific, Hemel Hempstead, UK) and 
stained with haematoxylin and eosin [16]. Images were digitally captured (at $\times 10$ magnification) using an inverted light microscope (Olympus BX41, Olympus, Southend-on-Sea, UK). One slide was prepared for each rat and five fields of view per slide (whole cells only in field of view) were analysed and quantified using Cell ${ }^{\wedge} \mathrm{P}$ software (Olympus, Shinjuku, Tokyo, Japan) ( $n=6$ rats per group).

Gene expression analysis Total RNA was extracted using Direct-zol RNA MiniPrep kits (Zymo Research, Irvine, CA, USA) with an inclusion of a DNAse digestion step. RNA purity and concentration was determined by spectrophotometric analysis (NanoDrop ND-1000, Thermo Scientific, UK), and RNA integrity was determined by agarose gel electrophoresis. cDNA was synthesised using a High-Capacity cDNA Reverse Transcription Kit (Applied Biosystems, Warrington, UK). Gene expression was assessed by quantitative (q)RT-PCR using custom designed primers (Sigma-Aldrich, Haverhill, UK and SYBR Green (Applied Biosystems; primer sequences are shown in Table 1). Analysis of melting curves confirmed the absence of primer dimers (StepOnePlus Real-Time PCR System, Applied Biosystems). Genomic rat DNA (Novagen, Madison, WI, USA) was serially diluted to generate standard curves. Several housekeeping genes were tested: Gapdh, Ppia and Ywhaz in epididymal fat; and Gapdh, Hprt, Nono, Ppia, Actb, Ywhaz, Rpl and Gusb in differentiated adipocytes. Expression of mRNA was normalised to Gapdh and Ywhaz expression because these genes had the least variable expression that was not affected by the maternal diet. Expression of the Cebpa gene (encoding CCAAT/enhancer-binding protein alpha) was determined using TaqMan gene expression assay ID Rn00560963_s1 and TaqMan reagents (Applied Biosystems).

miRNA expression analysis Putative miRNAs targeting the $3^{\prime}$ untranslated regions (UTRs) of Irs $1, P i k 3 c b$ (gene encoding the catalytic subunit of PI3K [p110ß]), Pik3rl (gene encoding the regulatory subunit of PI3K [p $85 \alpha]$ ) and Akt1 were identified using the TargetScan [17], microRNA.org [18], miRTarBase [19] and miRanda/mirSVR [20] tools. Candidate miRNAs were ranked according to their conservation across species, the strength of the predicted interaction, whether the mRNA target site was within a proximal or distal location, and their expression in WAT. Total RNA was reverse transcribed using a TaqMan MicroRNA Reverse Transcriptase Kit (Applied Biosystems) and miRNA-specific primers (TaqMan MicroRNA Assays): miR-19a-3p, ID 000395; miR-25-3p, ID 000403; miR-30a-5p, ID 000417; miR-93-5p, ID 001090; miR-126, ID 002228; miR-128a, ID 002216; miR-130a-3p, ID 000454; miR-145, ID 002278; miR-222, ID 002276; miR-301a-3p, ID 000528; miR-3203p, ID 002277; and miR-335-5p, ID 000546 (Applied Biosystems). qRT-PCR was performed using a TaqMan Universal PCR Master Mix No AmpErase UNG kit (Applied Biosystems). Standard curves were generated using serial dilutions of pooled sample cDNA.

Protein analysis Western Blotting was done as previously described [21]. Primary antibodies used were: anti-IRS-1 and anti-PI3K p $85 \alpha$ subunit (Millipore, Lake Placid, NY, USA); anti-Akt1, anti-Akt2, anti-phospho-Akt Ser473, antiphospho-extracellular signal-regulated kinases 1 and 2

Table 1 Primer sequences

\begin{tabular}{lll}
\hline Primer & Sequence (forward) & Sequence (reverse) \\
\hline Pparg & ACACAGACAAAACATCAGTGG & ACCATGCTCTGGGTCAACAG \\
Plin 1 & AGGGAGGGAACCCATGGAATA & TCTTCACGCTGCAAAGCAGA \\
Adipoq & CAAGGCCGTTCTCTTCACCT & CCCCATACACTTGGAGCCAG \\
Lep & CAAAACGTGCTGCAGATAGC & CCAGCAGATGGAGGAGGTC \\
Srebfl & AAGGCCATCGACTACATCCG & TGCTTTTGTGAGCACTTCGC \\
Cebpb & TTCCTTTCCGACCTCTTCGC & CACGTAACCGTAGTCGGACG \\
Cebpg & AGTTGAGTGTGGCCTTCTCG & CGACAGCTTGCTCATTTGGG \\
Insr & TGCCCACCACCCTACTATCA & GTATAGCCAGACGGGCACTC \\
Irs 1 & TGGCAGTGAGGATGTGA & GGATGC TCCCCC TAGAT \\
Pik3cb & ATGGCAGACACCCTTGACAT & GGTAGCTTCCCGGGGTACTT \\
Pik3r1 & AGGGGTACCAGTACAGAGCG & GTCAGGATGTCCCCCAAGTG \\
A $t 1$ & CATGGAGTGTGTGGACAGTGA & GATGATCCATGCGGGGCTTC \\
A $t 2$ & TGGAGCTCTGTTAGCACCGT & CAGTTCCGAGCTTGAGTGCC \\
Prkcz & CTAGCCATGGCCGGAGTG & GTCCATCTTGGGGTCGGTC \\
Slc $2 a 4$ & GGCCGGGACACTATACCCTA & AGAGCCGATCTGCTGGAAAC \\
Gapdh & CAGGGCTGCCTTCTCTTGTG & GATGGTGATGGGTTTCCCGT \\
Ywraz & GGCAGAGCGATACGATGACA & AAGATGACCTACGGGCTCCT \\
\hline
\end{tabular}


(ERK1/2) Thr202/Tyr204, anti-phospho c-Jun N-terminal kinases 1 and 2 (JNK1/2) Thr183/Tyr185 and anti-phospho-p38 mitogen-activated protein kinase (MAPK) Thr180/Tyr182 (Cell Signaling, Danvers, MA, USA); anti-insulin receptor beta (IR $\beta)$, anti-PI3K p110 $\beta$ subunit, anti-protein kinase $\mathrm{C} \zeta$ (PKC $\zeta$ ) and anti-suppressor of cytokine signalling 1 (SOCS1; Santa Cruz Biotechnology, Heidelberg, Germany); and antiGLUT4 (Abcam, Cambridge, UK). Peroxidase-conjugated anti-rabbit or anti-mouse secondary antibodies were used (Jackson ImmunoResearch, Stratech, Newmarket, UK).

Isolation and in vitro differentiation of primary preadipocytes Epididymal fat was dissected and collected into Hanks Basic Salt Solution (HBSS; all chemicals from SigmaAldrich). For each pre-adipocyte preparation, $7 \mathrm{~g}$ fat was chopped, placed into $30 \mathrm{ml}$ digestion solution (45 mg collagenase type II and $675 \mathrm{mg}$ BSA in HBSS), shaken at $180 \mathrm{rev} / \mathrm{min}$ for $40-50 \mathrm{~min}$ at $37^{\circ} \mathrm{C}$, strained through a $100 \mu \mathrm{m}$ mesh (BD Falcon, Tewksbury, MA, USA) and placed on ice for $20 \mathrm{~min}$. The upper layer containing mature adipocytes was then removed. The top two-thirds was mixed with growth medium (high glucose DMEM supplemented with $10 \%$ newborn calf serum, $1 \%$ penicillin-streptomycin and $200 \mu \mathrm{mol} / 1$ L-glutamine). The stromal-vascular fraction was centrifuged and re-suspended in growth medium. Following a second centrifugation, the pellets were re-suspended in $5 \mathrm{ml}$ erythrocyte lysis buffer (150 mmol/1 ammonium chloride, $10 \mathrm{mmol} / \mathrm{l}$ potassium bicarbonate, $0.1 \mathrm{mmol} / 1$ EDTA, $\mathrm{pH} \mathrm{8.0)} \mathrm{for} 5 \mathrm{~min}$. Cells were pelleted, re-suspended in growth medium, counted and plated at a density of $1 \times 10^{4}$ per $\mathrm{cm}^{2}$ into $75 \mathrm{ml}$ flasks. The culture medium was supplemented with an adipogenic cocktail (30 $\mu \mathrm{mol} / 1$ insulin [Actrapid, Novo Nordisk, Gatwick, UK], $150 \mu \mathrm{mol} / 1$ sodium ascorbate) daily for the first 3 days and then every other day until harvesting on day 11.

Statistical analysis Data were analysed using the Student's $t$ test and corrected for multiple comparisons (Holm-Šidák method; GraphPad Prism 6, La Jolla, CA, USA). The adipocyte area is presented as median values (interquartile range [IQR]) and was analysed using the Mann-Whitney $U$ test. SOCS1 data were analysed using unpaired t-tests with Welch's correction. Protein content, protein phosphorylation and mRNA expression data are the percentage of control \pm SEM; all other data are the means \pm SEM. $n$ values refer to the number of litters. A $p$ value of $<0.05$ was considered statistically significant.

\section{Results}

Body weight, fat mass, fat deposition and metabolic profile We confirmed previous findings of a significant lifelong reduction in body weight and length of male LP offspring compared with controls (Table 2) [22]. The retroperitoneal fat pad mass was significantly reduced in LP offspring rats in both absolute and relative terms, while no significant difference was observed in the epididymal or intra-abdominal fat pad masses (Table 2). Total fat mass (i.e. the combined weight of retroperitoneal, epididymal and intra-abdominal fat pads) was significantly reduced in LP offspring animals compared with controls in both absolute $(56.5 \pm 3.5 \mathrm{~g}$ vs $76.8 \pm 5.7 \mathrm{~g}$, $p<0.01)$ and relative terms $(6.8 \% \pm 0.2 \%$ vs $8.1 \% \pm 0.4 \%$, $p<0.01)$. To investigate WAT distribution, we calculated the weight of individual fat pads as the percentage of total fat mass (Fig. 1). Two-way ANOVA revealed an interaction between maternal diet and fat distribution $(p=0.00001)$, with the LP offspring group showing a relative increase in epididymal $(p<0.01)$ and reduction in retroperitoneal $(p<0.001)$ fat pad masses compared with controls (Fig. 1).

Fasting glucose, insulin, leptin, cholesterol, triacylglycerol, HDL and NEFA levels were not different between the two groups (Table 3 ). This supports our strategy of investigating molecular changes that precede any differences in metabolic status.

Morphology of epididymal WAT The median adipocyte area was reduced in epididymal WAT from LP offspring compared with controls $\left(387.2 \mu \mathrm{m}^{2}\right.$ [IQR 176.6-696.5 $\mu^{2}$ ] vs $600.7 \mu \mathrm{m}^{2}$ [IQR 260.2-1,004.0 $\mu^{2}$ ], $p<0.001$; MannWhitney $U$ test). Epididymal WAT from LP offspring contained a higher percentage of small adipocytes compared with controls ( $p<0.001$; Fig. 2). However, lipid content was comparable between the two groups (controls: $70.1 \% \pm 2.0 \%$ vs LP offspring: $72.3 \% \pm 2.1 \%$ of total weight of a fat pad).

Insulin signalling proteins in epididymal fat No differences in mRNA expression were observed for key insulin signalling molecules in epididymal WAT (Fig. 3a). IR $\beta$ protein levels were not different between the two groups (Fig. 3b). However,

Table 2 Body weight and body length, absolute and relative fat pad weights

\begin{tabular}{lll}
\hline Characteristic & Control offspring & LP offspring \\
\hline Body weight (g) & $946 \pm 47$ & $813 \pm 30^{*}$ \\
Body length (cm) & $291 \pm 2$ & $281 \pm 2 * *$ \\
Epididymal fat (g) & $19.8 \pm 0.9$ & $17.5 \pm 0.9$ \\
Epididymal fat (\% BW) & $2.1 \pm 0.1$ & $2.2 \pm 0.0$ \\
Retroperitoneal fat (g) & $50.4 \pm 4.9$ & $32.7 \pm 2.6^{* *}$ \\
Retroperitoneal fat (\% BW) & $5.3 \pm 0.4$ & $3.9 \pm 0.2^{* *}$ \\
Intra-abdominal fat (g) & $6.6 \pm 0.4$ & $6.2 \pm 0.4$ \\
Intra-abdominal fat (\% BW) & $0.7 \pm 0.0$ & $0.8 \pm 0.0$ \\
\hline
\end{tabular}

Data are means \pm SEM

${ }^{*} p<0.05,{ }^{* *} p<0.01$

$\mathrm{BW}$, body weight 


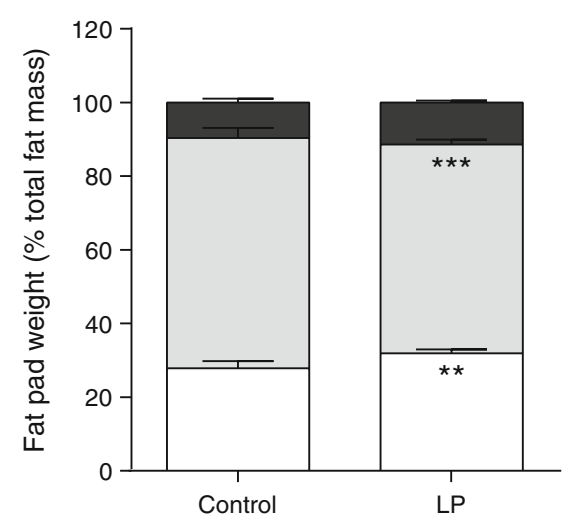

Fig. 1 Effect of maternal low protein diet on WAT distribution in male 14-month-old offspring. The weight of epididymal (white), retroperitoneal (light grey) and intra-abdominal fat (black) in controls $(n=9)$ and LP offspring $(n=12)$ rats are presented as the percentage of total fat mass \pm SEM. Data were analysed using two-way ANOVA followed by Duncan's post hoc testing. ${ }^{* *} p<0.01,{ }^{* * *} p<0.001$

the protein content of downstream signalling molecules including IRS1 and the PI3K catalytic subunit $\mathrm{p} 110 \beta$ were significantly reduced in epididymal WAT from LP offspring $(p<0.05$ for both). Levels of PI3K regulatory subunit $\mathrm{p} 85 \alpha$ were comparable to those of controls (Fig. 3b). Akt1 protein was significantly reduced in LP offspring $(p<0.05)$, while Akt2 remained unchanged. Phosphorylated Akt (Ser473) and $\mathrm{PKC} \zeta$ protein levels were also decreased in LP offspring (both $p<0.05$ ), while levels of GLUT4 were comparable to those of controls (Fig. 3a). These findings are consistent with WAT insulin resistance in the LP offspring.

Levels of key insulin signalling proteins are reduced in in vitro differentiated pre-adipocytes from LP offspring There was no difference in the mRNA level of any of the key insulin signalling molecules studied in adipocytes derived from LP offspring and control pre-adipocytes after in vitro differentiation (Fig. 4a). However, levels of IR $\beta(p<0.05)$, IRS1 $(p<0.05)$, PI3K p110 $\beta(p<0.05)$ and PI3K p85 $\alpha$ $(p<0.01)$ proteins were significantly reduced in differentiated adipocytes derived from LP offspring compared with controls

Table 3 Metabolic profile of 14-month-old control and LP offspring

\begin{tabular}{lll}
\hline Metabolic factor & Control offspring & LP offspring \\
\hline Glucose $(\mathrm{mmol} / \mathrm{l})$ & $5.5 \pm 0.2$ & $5.5 \pm 0.2$ \\
Insulin $(\mathrm{pmol})$ & $270 \pm 28$ & $248 \pm 31$ \\
Leptin $(\mathrm{ng} / \mu \mathrm{l})$ & $39 \pm 7$ & $38 \pm 7$ \\
Cholesterol $(\mathrm{mmol} / \mathrm{l})$ & $6.23 \pm 1.24$ & $5.43 \pm 0.55$ \\
Triacylglycerols $(\mathrm{mmol} / \mathrm{l})$ & $0.7 \pm 0.2$ & $0.5 \pm 0.1$ \\
HDL $(\mathrm{mmol} / \mathrm{l})$ & $1.70 \pm 0.44$ & $1.37 \pm 0.16$ \\
NEFA $(\mu \mathrm{mol} / \mathrm{l})$ & $575 \pm 62$ & $618 \pm 46$ \\
\hline
\end{tabular}

Data are the means \pm SEM. Due to the unequal variances, data for triacylglycerols was $\log _{10}$-transformed prior to the analysis
(Fig. 4b). Despite the increase in Akt1 protein level $(p<0.05)$ and no change in Akt2 protein level, Akt Ser473 phosphorylation was also reduced $(p<0.05)$ in in vitro differentiated adipocytes from LP offspring (Fig. 4b). This suggests reduced insulin action in programmed adipocytes after in vitro differentiation.

Effect of maternal protein restriction on pre-adipocyte differentiation We next investigated whether the reduced protein content and phosphorylation of key insulin signalling proteins in differentiated adipocytes from LP offspring were related to differences in the levels of differentiation markers. As differentiation is mainly driven by transcriptional mechanisms, we measured the mRNA expression of key differentiation marker genes: Pparg, Plin1, Adipoq, Lep, Srebf1 (gene encoding sterol regulatory element-binding protein 1), Cebpa, Cebpb and Cebpg. We did not observe differences in the mRNA levels of any of these markers (Table 4), suggesting that impaired insulin signalling and action in LP offspring adipocytes is not related to differences in differentiation.

MiRNA expression in primary pre-adipocytes after in vitro differentiation As a maternal low protein diet had profound effects on the levels of IRS1, PI3K p $85 \alpha$ and p $110 \beta$ subunits, and Akt1 without changing the corresponding mRNA levels, we investigated the mechanisms underlying these effects with a particular focus on the potential role of miRNAs. We used a candidate approach to examine the expression of miRNAs with complementary binding seed sequences in the 3'UTR of four genes: Irs 1, Pik3cb, Pik3r1 and $A k t 1$. For each gene, a panel of miRNAs was selected following bioinformatic assessment. None of the miRNAs selected for potentially regulating $I r s 1, P i k 3 r 1$ or $A k t 1$ were upregulated in LP offspring (Fig. 5). Of the four miRNAs selected for $P i k 3 c b$, expression of two, miR-25-3p and miR$130 \mathrm{a}-3 \mathrm{p}$, was reduced ( $p<0.05$ and $p<0.001$, respectively) in in vitro differentiated adipocytes from LP offspring (Fig. 5).

Markers of protein degradation in in vitro differentiated pre-adipocytes from LP offspring Maternal protein restriction led to a significant increase in IRS1 Ser307 phosphorylation in differentiated adipocytes from LP offspring $(p<0.01)$, while IRS1 Tyr612 phosphorylation was reduced $(p<0.05$; Fig. 6a). The ratio of Ser307 phosphorylated IRS1 to total IRS1 was increased in differentiated adipocytes from LP offspring compared with controls ( $p<0.001$; Fig. $6 \mathrm{~b}$ ). There was no difference in ERK1/2 Thr202/Tyr204 phosphorylation or JNK1/2 Thr183/Tyr185 phosphorylation between the groups; however, p38 MAPK Thr180/Tyr182 phosphorylation was increased in differentiated adipocytes from LP offspring animals $(p<0.05$; Fig. $6 c)$. SOCS1 protein content was also increased in differentiated adipocytes from LP offspring compared with controls $(p<0.05$; Fig. $6 \mathrm{~d})$. 
a

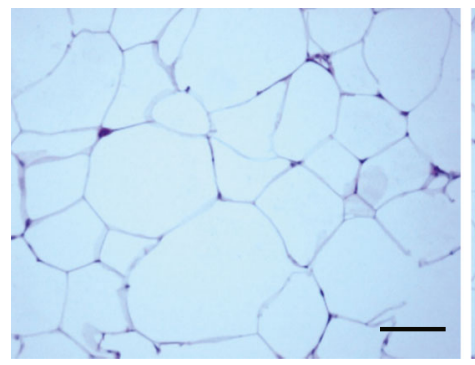

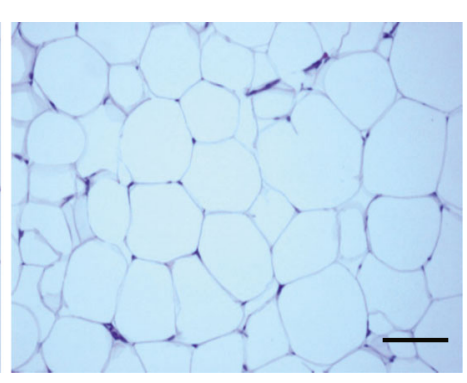

b

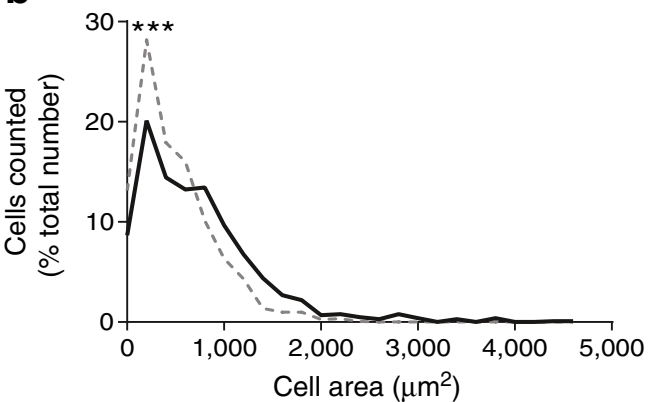

Fig. 2 Effect of maternal low protein diet on the epididymal adipose tissue morphology of 14-month-old offspring rats. (a) Representative histological images from controls (left) and LP offspring (right). Scale bar represents $100 \mu \mathrm{m}$. (b) Adipocyte area frequency distribution of control (black line) and LP offspring (dashed line) rats. Median adipocyte

\section{Discussion}

The present study investigated the mechanisms involved in mediating the effects of suboptimal early nutrition on the development of peripheral insulin resistance. Significant changes in body fat distribution, adipocyte size and the levels and phosphorylation status of key insulin signalling proteins were observed in WAT from adult male rats exposed to maternal protein restriction during gestation and lactation. Moreover, the molecular phenotype of impaired insulin signalling was area within epididymal adipose tissue differed between LP offspring and controls ( $p<0.001$, Mann-Whitney $U$ test; $n=6$ rats per group). One slide was prepared from each rat and five fields of view were analysed per slide. ${ }^{* * *} p<0.001$

retained in isolated pre-adipocytes from LP offspring after in vitro differentiation. Importantly, as LP offspring were normoglycaemic and normoinsulinaemic at the time of study, these changes are not a consequence of metabolic dysfunction but are likely to contribute to the later development of whole body insulin resistance and type 2 diabetes.

We observed that maternal protein restriction led to a reduction in total visceral WAT (intra-abdominal, retroperitoneal and epididymal fat depots combined) and altered the distribution of fat within the body in the offspring. In LP offspring, relative fat

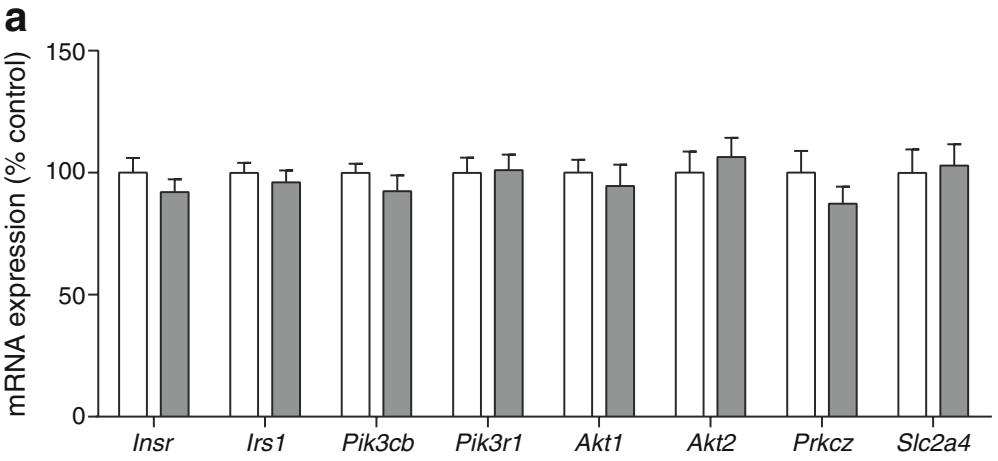

b

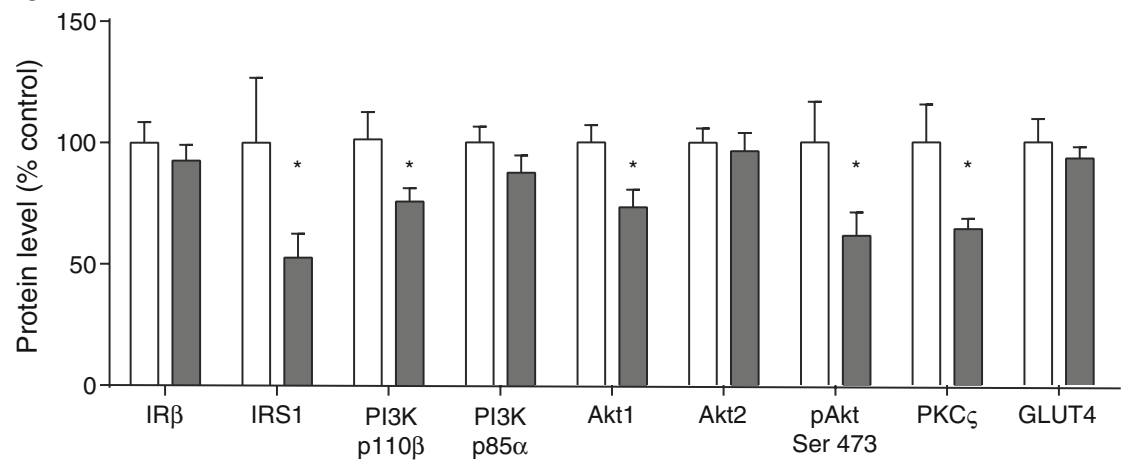

C

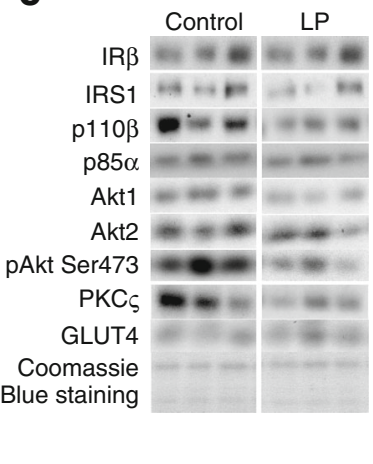

Fig. 3 Effect of maternal low protein diet on the expression of insulin signalling molecules in epididymal WAT of 14-month-old male offspring rats. (a) mRNA levels, (b) protein content and phosphorylation and (c) representative protein blots. (a, b) Data are the percentage of control values $\pm \mathrm{SEM} ; n=8$ for controls, $n=10$ for LP offspring; ${ }^{*} p<0.05$. White bars, control; grey bars, LP. Slc2a 4 encodes GLUT4 


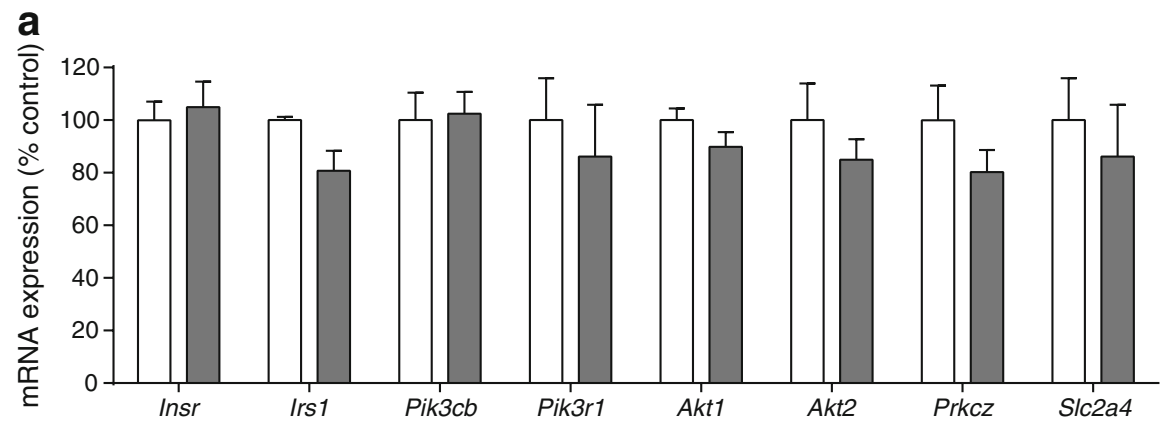

b

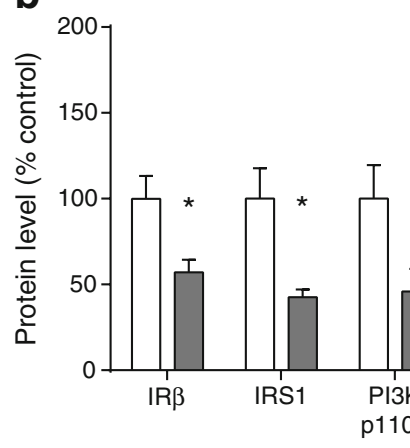

Fig. 4 Effect of maternal low protein diet on the levels of key insulin signalling molecules in differentiated adipocytes from offspring rats. (a) mRNA levels in controls $(n=8)$ and LP offspring $(n=10)$. (b) Protein

deposition within the epididymal depot was increased, while deposition into the retroperitoneal depot was reduced. Changes in body fat distribution have been observed in young, lean normoglycaemic LBW men, and may contribute to the development of impaired glucose tolerance and type 2 diabetes in developmentally programmed individuals [23]. Altered fat deposition may lead to alterations in sympathetic drive and rates of lipolysis because these vary between different fat depots [10]. Increased rates of lipolysis have been found in adipocytes isolated from 3-month-old LP offspring rats and LBW men [24-26]. However, the full impact of altered fat distribution in LP offspring on metabolic phenotype remains to be determined.

Table 4 Levels of differentiation marker mRNAs in in vitro differentiated adipocytes

\begin{tabular}{llll}
\hline mRNA & Control offspring & LP offspring & $p$ value \\
\hline Pparg & $0.014 \pm 0.001$ & $0.012 \pm 0.001$ & 0.22 \\
Plin1 & $0.181 \pm 0.033$ & $0.169 \pm 0.046$ & 0.81 \\
Adipoq & $1.550 \pm 0.300$ & $1.753 \pm 0.416$ & 0.72 \\
Lep & $0.140 \pm 0.027$ & $0.109 \pm 0.016$ & 0.30 \\
Srebf1 & $0.670 \pm 0.138$ & $0.497 \pm 0.101$ & 0.31 \\
Cebpa & $0.084 \pm 0.014$ & $0.060 \pm 0.012$ & 0.12 \\
Cebpb & $1.389 \pm 0.096$ & $1.176 \pm 0.095$ & 0.15 \\
Cebpg & $0.031 \pm 0.003$ & $0.026 \pm 0.002$ & 0.19 \\
\hline
\end{tabular}

Data are the means \pm SEM

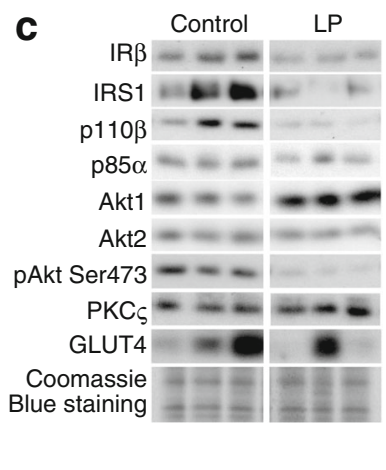

content and phosphorylation ( $n=6$ per group). (c) Representative protein blots. (a, b) Data are the percentage of control values \pm SEM; ${ }^{*} p<0.05$, $*^{* *} p<0.01$. White bars, control; grey bars, LP. Slc2a4 encodes GLUT4

We have previously shown that 3-month-old LP offspring have reduced adipocyte size. This was associated with increased miR-483 expression in both rodents and humans and led to a reduction in the expandability of adipose tissue [7]. Here, we showed that reduced adipocyte size is retained in 14month-old LP offspring rats and that a higher percentage of small adipocytes can be found in epididymal WAT of these animals compared with controls. An increased proportion of small adipocytes and a corresponding deficit in large adipocytes have been associated with insulin resistance [27].

Impairments in insulin signalling including decreased IRS1 protein content, reduced Akt Ser473 phosphorylation, impaired insulin-stimulated PI3K activity and impaired glucose transport have been observed in adipocytes from individuals with a predisposition toward type 2 diabetes (i.e. with at least two first-degree relatives diagnosed with diabetes) [28]. A similar pattern of abnormalities can be detected in adipocytes from diabetic patients and LBW young men [5]. Here, we show reduced levels of IRS1, the p110 $\beta$ catalytic subunit of PI3K, Akt1 and PKC $\zeta$, and decreased Akt Ser473 phosphorylation in the epididymal fat of LP offspring. Importantly, we demonstrate that the reduction in key insulin signalling proteins (IR $\beta$, IRS1, PI3K p $85 \alpha$ and $\mathrm{p} 110 \beta$ subunits and Akt1) and decreased Akt Ser473 phosphorylation is retained in isolated primary pre-adipocytes from LP offspring after in vitro differentiation. This strongly suggests that cell-autonomous mechanism(s) retained throughout the life-course underlie the programmed phenotype. These are maintained following 
Fig. 5 The effect of maternal low protein diet on expression of miRNAs targeting the mRNA 3' UTRs of key insulin signalling genes in differentiated adipocytes from offspring rats. (a) $\operatorname{Irs} 1$, (b) Pik3cb, (c) Pik3r1, (d) Akt1. Data are the percentage of control values \pm SEM; $n=8$ controls, $n=10$ LP offspring; $* p<0.05$, $* * * p<0.001$. White bars, control; grey bars, LP a

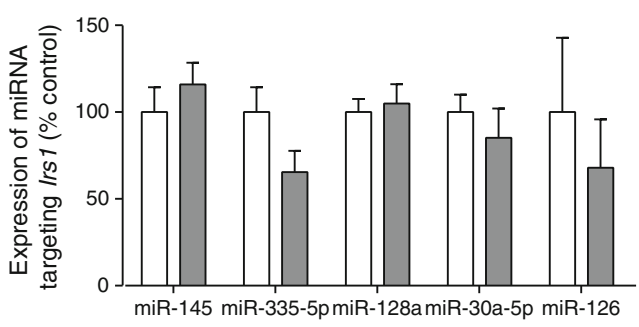

b

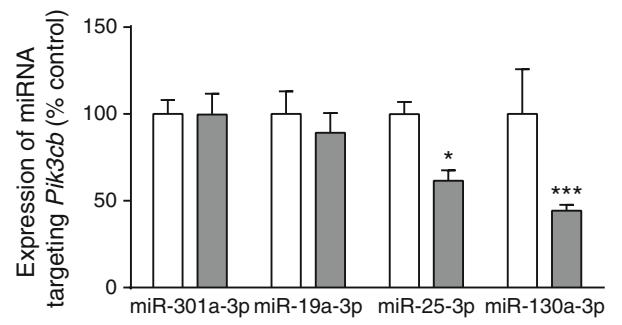

C

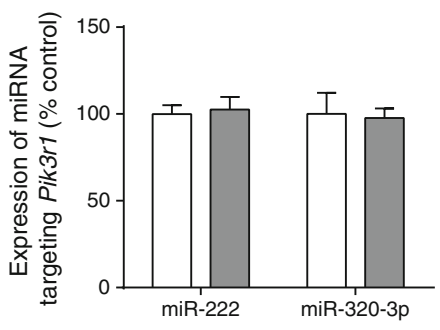

d

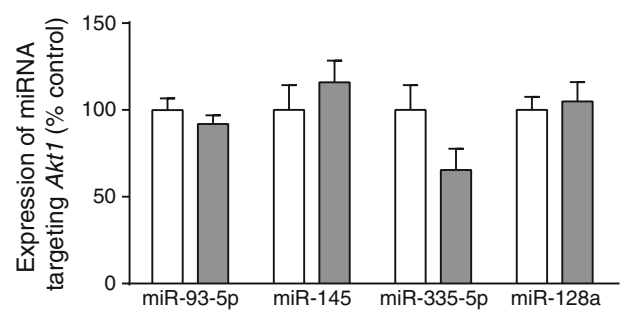

a

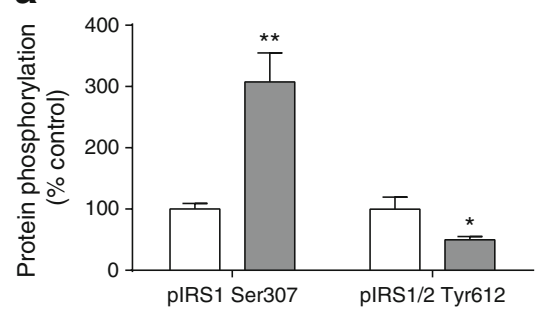

b
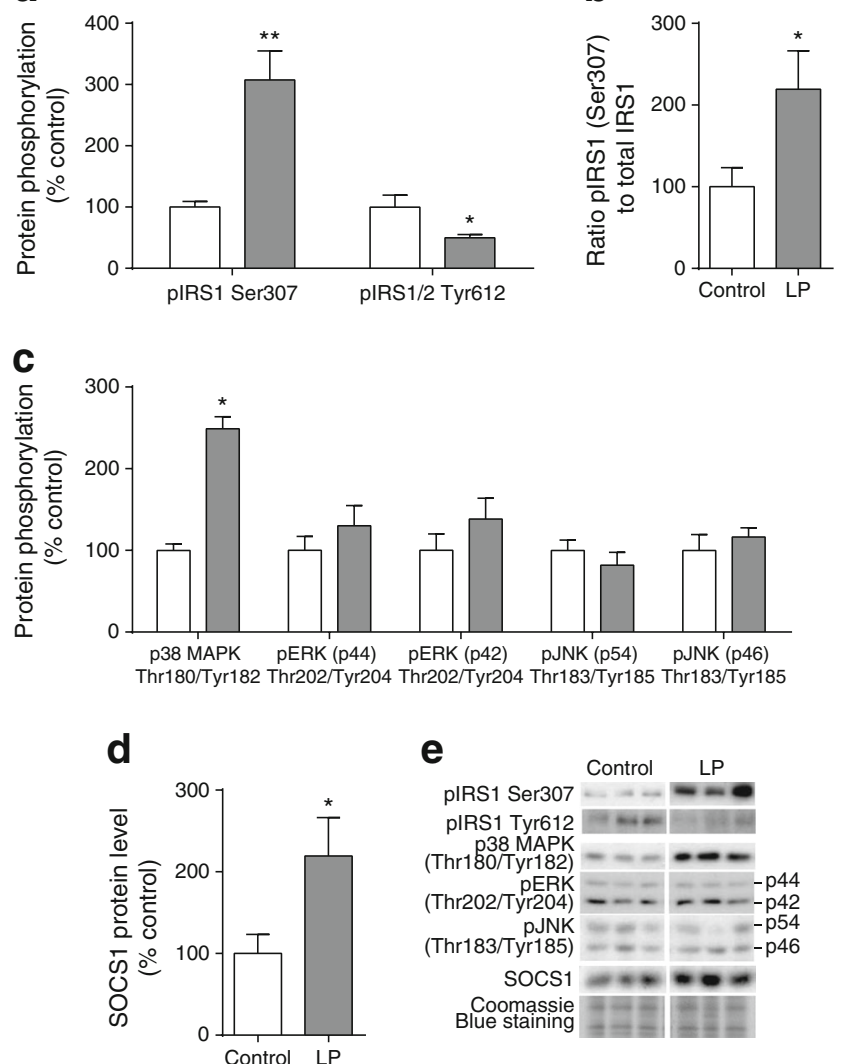

e

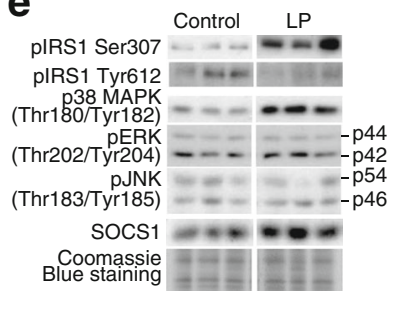

Fig. 6 The effects of maternal low protein diet on levels of markers of protein degradation in differentiated adipocytes from offspring rats. (a) IRS1 Ser307 and Tyr612 phosphorylation (pIRS1, phospho-IRS1), (b) ratio of Ser307 pIRS1 to total IRS1, (c) MAP kinase phosphorylation and (d) SOCS1 protein levels. (e) Representative protein blots. Data are the percentage of control values $\pm \mathrm{SEM} ; n=6$ per group. White bars, control; grey bars, LP. pERK, phospho-ERK; pJNK, phospho-JNK multiple rounds of cell division and differentiation in a controlled environment. As individuals with heritable predisposition to diabetes, LBW humans, LP offspring rats and mice exposed to maternal obesity during gestation and lactation had similar impairments in insulin signalling proteins in WAT (with IRS1 downregulation in mice also shown to be regulated in a cell-autonomous manner), common pathways may be initiated in response to a range of suboptimal early conditions that may drive the development of peripheral insulin resistance $[2,5,8,28]$.

Although until recently research in the programming field has been primarily directed at understanding mechanisms mediating changes in gene expression at a transcriptional level, changes in the levels of multiple proteins are seen without alterations in the corresponding mRNA transcript levels [7, $8,12]$. Our finding in LP offspring of impaired insulin signalling protein levels in both WAT and adipocytes in vitro differentiated without changes in the corresponding mRNA levels implies that the mechanisms underlying these programmed changes occur at the post-transcriptional level, and involve changes in protein synthesis and/or degradation.

To investigate the cell-autonomous mechanisms that may mediate the effects of maternal nutrition on susceptibility to developing peripheral insulin resistance in WAT in the offspring, we studied miRNAs implicated in regulating the synthesis of programmed insulin signalling proteins. MiRNAs repress mRNA translation by binding to specific sequences within the $3^{\prime} \mathrm{UTR}$ s of target mRNAs. Although numerous miRNAs have been implicated in the pathogenesis of insulin resistance and type 2 diabetes [29], information on which miRNAs may be involved in developmental programming in WAT is scarce. Recently, a programmed increase in miR126, was shown to repress translation of its $I r s 1$ target in WAT 
from mice exposed to maternal overnutrition in early life [8]. Here, we used a candidate approach to identify miRNAs that could potentially influence the translation of Irs 1, Pik3 $c b$, Pik3rl and Akt 1 mRNAs. However, none of the candidate miRNAs (including miR-126) were upregulated in adipocytes that were differentiated in utero in response to maternal low protein diet. Therefore, none of the changes in the levels of insulin signalling molecules could be explained by upregulation of these miRNAs. This implies that: (1) altered levels of these proteins are not mediated by the candidate miRNAs studied; (2) other unidentified miRNAs play a role; or (3) other post-transcriptional mechanisms are involved.

In the current study, we observed increased serine phosphorylation and decreased tyrosine phosphorylation in IRS1 in in vitro differentiated adipocytes from LP offspring. Although tyrosine phosphorylation is required to generate the insulin signal, serine phosphorylation occurs before, during and after insulin stimulation [30]. Increased IRS1 phosphorylation at serine 307 (rodents)/312 (humans) has been reported in insulin resistant states $[9,31]$ and nutritionally programmed animals [21]. One mechanism through which IRS1 can become phosphorylated at Ser307 involves activation of JNK $1 / 2$, a key component of the stress and inflammatory response pathway [32]. However, we observed no differences in JNK1/2 Thr183/Tyr185 phosphorylation in differentiated adipocytes from LP offspring and controls, suggesting that other cell-autonomous mechanism(s) must be involved in upregulating IRS1 Ser307 phosphorylation. Prolonged IRS1 serine/ threonine phosphorylation following chronic insulin stimulation leads to its targeted degradation by the proteasome [33, 34]. Increased IRS1 ubiquitination and its subsequent degradation have been reported in a mouse model of type 2 diabetes (TALLYHO/Jng mice) and were associated with an increase in SOCS1 levels [9]. SOCS proteins are important mediators of IRS1 degradation and target IRS1 via interaction with the SOCS box motif [35]. Increased levels of SOCS1 in differentiated adipocytes from LP offspring animals suggests that lower IRS1 protein levels in these animals may be at least partly caused by SOCS1-mediated degradation of IRS1.

We investigated whether the other two MAPKs could play a role in the cell-autonomous programming of insulin signalling proteins. There was no difference in ERK Thr202/Tyr204 phosphorylation in differentiated adipocytes from LP offspring and controls. However, p38 MAPK Thr180/Tyr182 phosphorylation was significantly upregulated in differentiated adipocytes from LP offspring animals. Enhanced basal p38 MAPK activation has also been reported in adipocytes from type 2 diabetic patients [36]. An in vitro study of isolated skeletal muscle strips showed that low levels of oxidative stress lead to increased p38 MAPK Thr180/Tyr182 phosphorylation which was associated with decreased IRS1 protein levels and inhibition of insulin-stimulated Akt Ser473 phosphorylation [37].
In conclusion, we have demonstrated that a maternal low protein diet during gestation and lactation leads to altered adipose tissues distribution and insulin resistance in the WAT of male offspring. This is associated with reductions in protein levels and phosphorylation of key insulin signalling molecules in WAT. This phenotype was retained in primary in vitro differentiated adipocytes, suggesting that cellautonomous mechanism(s) are responsible. These findings bring us a step closer to understanding the mechanisms that mediate the effects of suboptimal early nutrition on the risk of developing type 2 diabetes.

Acknowledgements We thank K. Phillips, A. Wayman and F. Ong for technical assistance (the Phenomics Laboratory, University of Cambridge). Lipid profiling was performed by the Core Biochemical Assay Laboratory, Cambridge University Hospitals NHS Foundation Trust, Cambridge, UK.

Funding This work was supported by Diabetes UK (MSM-G; no. 12/ 0004508), the British Heart Foundation (SEO; no. FS/09/029/27902) and the UK Medical Research Council (SEO; no. MC_UU_12012/4)

Duality of interest statement The authors declare that there is no duality of interest associated with this manuscript.

Contribution statement All authors contributed to the study conception and design; MSM-G and DSF-T acquired the data; MSM-G analysed the data and wrote the manuscript; all authors interpreted the data, revised the manuscript critically for important intellectual content and gave final approval of the version to be published. SEO is responsible for the integrity of the work as a whole.

Open Access This article is distributed under the terms of the Creative Commons Attribution 4.0 International License (http:// creativecommons.org/licenses/by/4.0/), which permits unrestricted use, distribution, and reproduction in any medium, provided you give appropriate credit to the original author(s) and the source, provide a link to the Creative Commons license, and indicate if changes were made.

\section{References}

1. Martin-Gronert MS, Ozanne SE (2007) Experimental IUGR and later diabetes. J Intern Med 261:437-452

2. Ozanne SE, Dorling MW, Wang CL, Nave BT (2001) Impaired PI 3-kinase activation in adipocytes from early growth-restricted male rats. Am J Physiol Endocrinol Metab 280:E534-E539

3. Ozanne SE, Olsen GS, Hansen LL et al (2003) Early growth restriction leads to down regulation of protein kinase $\mathrm{C}$ zeta and insulin resistance in skeletal muscle. J Endocrinol 177:235-241

4. Ozanne SE, Jensen CB, Tingey KJ, Storgaard H, Madsbad S, Vaag AA (2005) Low birthweight is associated with specific changes in muscle insulin-signalling protein expression. Diabetologia 48:547552

5. Ozanne SE, Jensen CB, Tingey KJ et al (2006) Decreased protein levels of key insulin signalling molecules in adipose tissue from young men with a low birthweight: potential link to increased risk of diabetes? Diabetologia 49:2993-2999 
6. Jensen CB, Martin-Gronert MS, Storgaard H et al (2008) Altered PI3-kinase/Akt signalling in skeletal muscle of young men with low birth weight. PLoS One 3:e3738

7. Ferland-McCollough D, Fernandez-Twinn DS, Cannell IG et al (2012) Programming of adipose tissue miR-483-3p and GDF-3 expression by maternal diet in type 2 diabetes. Cell Death Differ 19:1003-1012

8. Fernandez-Twinn DS, Alfaradhi MZ, Martin-Gronert MS et al (2014) Downregulation of IRS-1 in adipose tissue of offspring of obese mice is programmed cell-autonomously through posttranscriptional mechanisms. Mol Metab 3:325-333

9. Wang Y, Nishina PM, Naggert JK (2009) Degradation of IRS1 leads to impaired glucose uptake in adipose tissue of the type 2 diabetes mouse model TALLYHO/Jng. J Endocrinol 203:6-7

10. Rosen ED, Spiegelman BM (2014) What we talk about when we talk about fat. Cell 156:20-44

11. Feng B, Zhang T, Xu H (2013) Human adipose dynamics and metabolic health. Ann N Y Acad Sci 1281:160-177

12. Berends LM, Fernandez-Twinn DS, Martin-Gronert MS, Cripps RL, Ozanne SE (2013) Catch-up growth following intra-uterine growth restriction programs an insulin resistant phenotype in adipose tissue. Int J Obes (Lond) 37(8):1051-1057

13. Tarry-Adkins J, Fernandez-Twinn D, Madsen R et al (2015) Coenzyme Q10 prevents insulin signalling dysregulation and inflammation prior to development of insulin resistance in male offspring of a rat model of poor maternal nutrition and accelerated postnatal growth. Endocrinology 156(10):3528-3537

14. Chen JH, Martin-Gronert MS, Tarry-Adkins J, Ozanne SE (2009) Maternal protein restriction affects postnatal growth and the expression of key proteins involved in lifespan regulation in mice. PLoS One 4:e4950

15. Folch J, Lees M, Sloane Stanley GH (1957) A simple method for the isolation and purification of total lipides from animal tissues. $\mathrm{J}$ Biol Chem 226:497-509

16. Berry R, Church CD, Gericke MT, Jeffery E, Colman L, Rodeheffer MS (2014) Imaging of adipose tissue. In: MacDougald OA (ed) Methods in enzymology. Methods of adipose tissue biology, part 1, vol 537. Academic Press, Elsevier, London, pp 58-60

17. Grimson A, Farh KK, Johnston WK, Garrett-Engele P, Lim LP, Bartel DP (2007) MicroRNA targeting specificity in mammals: determinants beyond seed pairing. Mol Cell 27:91-105

18. Betel D, Wilson M, Gabow A, Marks DS, Sander C (2008) The microRNA.org resource: targets and expression. Nucleic Acids Res 36(Database Issue):D149-D153

19. Hsu S-D, Tseng Y-T et al (2014) miRTarBase update 2014: an information resource for experimentally validated miRNA-target interactions. Nucleic Acids Res 42:D78-D85

20. Betel D, Koppal A, Agius P, Sander C, Leslie C (2010) Comprehensive modeling of microRNA targets predicts functional non-conserved and non-canonical sites. Genome Biol 11:R90

21. Martin-Gronert MS, Fernandez-Twinn DS, Poston L, Ozanne SE (2010) Altered hepatic insulin signaling in male offspring of obese mice. J Dev Orig Health Dis 1:184-191
22. Petry CJ, Dorling MW, Pawlak DB, Ozanne SE, Hales CN (2001) Diabetes in old male offspring of rat dams fed a reduced protein diet. Int J Exp Diabetes Res 2:139-143

23. Rasmussen EL, Malis C, Jensen CB et al (2005) Altered fat tissue distribution in young adult men who had low birth weight. Diabetes Care 28:151-153

24. Ozanne SE, Wang CL, Dorling MW, Petry CJ (1999) Dissection of the metabolic actions of insulin in adipocytes from early growthretarded male rats. J Endocrinol 162(2):313-319

25. Petry CJ, Dorling MW, Wang CL, Pawlak DB, Ozanne SE (2000) Catecholamine levels and receptor expression in low protein rat offspring. Diabet Med 17(12):848-853

26. Højbjerre L, Alibegovic AC, Sonne MP (2011) Increased lipolysis but diminished gene expression of lipases in subcutaneous adipose tissue of healthy young males with intrauterine growth retardation. J Appl Physiol (1985) 111:1863-1870

27. McLaughlin T, Sherman A, Tsao P et al (2007) Proportion of small adipose cells in insulin-resistant vs insulin-sensitive obese individuals implicates impaired adipogenesis. Diabetologia 50:1707-1715

28. Carvalho E, Jansson PA, Nagaev I, Wenthzel AM, Smith U (2001) Insulin resistance with low cellular IRS-1 expression is also associated with low GLUT4 expression and impaired insulin-stimulated glucose transport. FASEB J 15:1101-1103

29. Chen H, Lan HY, Roukos DH, Cho WC (2014) Application of microRNAs in diabetes mellitus. J Endocrinol 222(1):R1-R10

30. Copps KD, White MF (2012) Regulation of insulin sensitivity by serine/threonine phosphorylation of insulin receptor substrate proteins IRS1 and IRS2. Diabetologia 55:2565-2582

31. Fröjdö S, Vidal H, Pirola L (2009) Alterations of insulin signaling in type 2 diabetes: a review of the current evidence from humans. Biochim Biophys Acta 1792:83-92

32. Hirosumi J, Tuncman G, Chang L et al (2002) A central role for JNK in obesity and insulin resistance. Nature 420:333-336

33. Rice KM, Turnbow MA, Garner CW (1993) Insulin stimulates the degradation of IRS-1 in 3T3-L1 adipocytes. Biochem Biophys Res Commun 190:961-967

34. Sun XJ, Goldberg JL, Qiao LY, Mitchell JJ (1999) Insulin-induced insulin receptor substrate-1 degradation is mediated by the proteasome degradation pathway. Diabetes 48:1359-1364

35. Ueki K, Kondo T, Kahn CR (2004) Suppressor of cytokine signaling 1 (SOCS-1) and SOCS-3 cause insulin resistance through inhibition of tyrosine phosphorylation of insulin receptor substrate proteins by discrete mechanisms. Mol Cell Biol 24:5434-5446

36. Carlson CJ, Koterski S, Sciotti RJ, Poccard GB, Rondinone CM (2003) Enhanced basal activation of mitogen-activated protein kinases in adipocytes from type 2 diabetes: potential role of $\mathrm{p} 38 \mathrm{in}$ the downregulation of GLUT4. Diabetes 52:634-641

37. Diamond-Stanic MK, Marchionne EM, Teachey MK, Durazo DE, Kim JS, Henriksen EJ (2011) Critical role of the transient activation of p38 MAPK in the etiology of skeletal muscle insulin resistance induced by low-level in vitro oxidant stress. Biochem Biophys Res Commun 405:439-444 